LA W REN CE LIVERMORE NATIONAL LABORATORY

J. W. Banks

April 22, 2013 
This document was prepared as an account of work sponsored by an agency of the United States government. Neither the United States government nor Lawrence Livermore National Security, LLC, nor any of their employees makes any warranty, expressed or implied, or assumes any legal liability or responsibility for the accuracy, completeness, or usefulness of any information, apparatus, product, or process disclosed, or represents that its use would not infringe privately owned rights. Reference herein to any specific commercial product, process, or service by trade name, trademark, manufacturer, or otherwise does not necessarily constitute or imply its endorsement, recommendation, or favoring by the United States government or Lawrence Livermore National Security, LLC. The views and opinions of authors expressed herein do not necessarily state or reflect those of the United States government or Lawrence Livermore National Security, LLC, and shall not be used for advertising or product endorsement purposes.

This work performed under the auspices of the U.S. Department of Energy by Lawrence Livermore National Laboratory under Contract DE-AC52-07NA27344. 


\title{
Modularity and adaptability
}

\author{
J. W. Banks
}

The primary question I am addressing is how to construct simulation tools, in this case primarily PDE simulations, that can be made to efficiently use a future computer whose construction and parameters are not known exactly, but whose general construction can be inferred from current prevailing hardware trends. These trends inform our best estimates of the likely composition of such a next generation machine, and the outlook is that the computers of the future will be highly heterogenous, will penalize excessive communication (particularly with far away pieces of the machine), can likely be tuned to trade pure speed for reliability, and may experience frequent disruptions. In such an environment, my position as to what mathematical research should be performed to aid in this endeavor can be summed up as a need to stress the following points

- Increased modularity of the simulation tool

- Retention of original problem definition as much as possible

- Provide and analyze flexible alternatives.

\section{Increased modularity of the simulation tool}

The idea of increasing modularity is easily discussed by example. Consider a fluid-structure problem consisting of compressible fluid flow with an elastic body. Broadly speaking there are two primary simulation approaches for this FSI problem; partitioned and monolithic. In a partitioned approach the solvers for each piece are isolated from each other and coupled only through the interface. This is in contrast to a fully-coupled, monolithic approach where the entire system is advanced by a single unified solver, typically by an implicit method. In a partitioned solver each physical process is considered in isolation, so each solver constitutes a confined module. This kind of modularization provides distinct benefits from an emerging architecture standpoint in the form of reduced global communication, increase concurrency, simpler fault tolerance strategies, etc. One notable example that takes such a strategy to an extreme is the SETI@home initiative. Here a global calculation is divided into individual pieces that are farmed out and executed in isolation over a highly heterogenous network of machines, an expensive slow network, while dealing with the inevitable failures and faults in both networks and compute nodes (even to go as far as detecting maliciously manipulated results). This approach is successful precisely because of the modular nature of the underlying problem. In the context of PDE simulations there are further advantages to modularization in that one naturally introduces the possibility of local time stepping, which can be extremely beneficial for problems with large time-scale separation.

To be concrete these traits make modularization particularly attractive in the context of emerging computing architectures for several reasons. These techniques naturally increase algorithmic concurrency, reduce global synchronizations, and fit well within a modular fault tolerance strategy where global resilience is achieved by hardening isolated subcomponents and then combining these in a hierarchical manner. There are many examples of modular partitioning including approximate 
factored schemes, alternating-direction-implicit (ADI) methods, deferred correction, etc. Whatever the case, by introducing modularity, complex simulations are reduced to smaller subcomponents whose interconnectedness is more easily manipulated to take full advantage of a new generation of computer (for example overlapping communication with computation or restarting failed computations due to hardware interruptions, or incorporating local time stepping).

The challenge in this approach is primarily one of numerical stability, which is conveniently exactly the right match for applied math research. Significant new math research on developing decoupled modular strategies should be a priority and will likely lead to much better utilization of future machines. For example we have recently demonstrated that by using detailed numerical analysis, stable partitioned solvers can be created for compressible FSI problems. Our work is now pushing these results into incompressible FSI regimes and more. Other applications are likely to see similar benefit from a renewed push toward increased modularization.

\section{Retention of original problem definition as much as possible}

The primary point here is that it is useful to retain as much of the high-level description of the problem as possible. Higher level descriptions retain important information about the nature of the computation that can be used when mapping the simulation onto a new machine. For example it is extremely unlikely that the intent of an application is to solve the matrix equation $A x=b$. To be sure this will be a critical step in many simulation tools, but the set of solution strategies that may be appropriate depends significantly on the nature of the continuous problem. For example elliptic problems are likely to be able to take advantage of multigrid while hyperbolic problems may have trouble when treated with the same techniques. A Helmholtz problem may result in poor behavior from any iterative approach. In addition, if at the highest level we know that perhaps a Poisson solver is just a small piece of a larger Navier-Stokes solver, we can offset some intrinsic lack of scalability of multigrid by performing other useful work (grid generation, load rebalancing, etc ...) while coarse-grid approximations are being performed. The closer one stays to the real application of interest, the more information is known. This information can be very useful when optimizing in a holistic manner.

\section{Provide and analyze flexible alternatives}

Finally it seems likely that future machines will be highly heterogenous. As a result, different algorithms and/or different implementations of the same algorithm are likely to perform significantly different on various pieces of the machine. In order to obtain optimal performance it will be important to dynamically select the best fit for each part of the machine. Flexibility is key here, and math analysis should be performed to decide which combinations can be permitted and which cannot. For example it may be that some part of the machine is unresponsive for some time and the remainder of the computation needs data from the unresponsive piece to continue. Rather than hold up the whole calculation, we might use a low cost model (such as extrapolation) for some time and then synch back up at some later time. The stability implications of such an approach are clearly significant, and detailed numerical analysis is needed to understand the limits of the overall approach. 